\title{
Le concept de science de la science psychothérapeutique
}

\author{
Markus Erismann
}

\author{
Psychotherapie-Wissenschaft 9 (2) 18-19 2019 \\ www.psychotherapie-wissenschaft.info \\ CC BY-NC-ND \\ https://doi.org/10.30820/1664-9583-2019-2-18
}

Mots clés : notion de science, science de la psychothérapie, le subjectif, l'individuel, le contradictoire

La question du concept de science se pose dans une phase de la crise d'une science ou une phase où cette science n'a pas encore atteint l'état d'une science normale. Selon Fischer (2008), la science psychothérapeutique se trouve encore dans un état "pré-paradigmatique ». Bien qu'une pratique scientifique sans un concept de science soit possible, le concept de science sert à former et à clarifier la compréhension de soi d'une science particulière et constitue un élément de son cadre scientifique théorique. L'intention de mon article est la présentation et la discussion du concept de science de la science psychothérapeutique. Il soutient leurs efforts pour établir une indépendante et équivalente science à côté de la psychologie et de la psychiatrie.

Le général concept de science se distingue par la triade objectivité - contrôlabilité - intersubjectivité. Ces critères scientifiques, qui sont étroitement interdépendants, constituent la base d'un savoir relativement sûr. La science possède en outre un caractère de généralité. La science est orientée sur le général : elle cherche des régularités, des modèles et des lois. L'individuelle et le particulier sont classifiés dans le général. Un autre critère scientifique est la cohérence : il faut que les phrases scientifiques obéissent aux règles de la logique formelle qui ne permette pas des contradictions.

Ces critères appelés impliquent une triple exclusion : la subjectivité, l'individuelle et la contradiction sont exclues $\mathrm{du}$ processus scientifique. Le concept de science de la science psychothérapeutique contredit dans ce cadre le générale concept de science : elle inclue la subjectivité, se concentre sur l'individuelle et intègre des contradictions et ambivalences dans leur méthode et leurs concepts théoriques.

La réponse sur la question du concept de science d'une science spéciale dépend de la compréhension de soi de cette science. La compréhension de soi doit être concordante avec le concept de science qui est reconnu généralement. Cette correspondance est la condition pour être reconnu comme une science normale. Comment est - malgré les trois aspects appelés qui contredisent en apparence le générale concept de science - une concordance entre le concept de science de la science psychothérapeutique et le générale concept de science possible ?

La science psychothérapeutique intègre la subjectivité dans le processus scientifique : il s'agit de la per- ception de la propre expérience dans la situation psychothérapeutique par l'introspection. Un moment d'arbitraire adhère au subjectif qui, en science, doit généralement être exclu par l'objectivité. Dans la science psychothérapeutique, qui inclue la subjectivité, l'arbitraire peut minimiser en ce que la subjectivité est accompagné d'une réflexion critique. Cela garantit l'objectivité et l'intersubjectivité.

La psychothérapie se concentre sur l'individualité du sujet particulier et le caractère unique de la relation entre le client et le psychothérapeute. Néanmoins, la science psychothérapeutique dépend aussi des généralisations. Afin de rendre justice à l'individualité, la recherche scientifique doit partir de cas individuels pour pouvoir ensuite découvrir au moyen de comparaison des structures plus générales. Dans ces structures, l'expérience thérapeutique est conservée et réfléchie en ce qui concerne des concepts théoriques.

La relation psychothérapeutique est déterminée par une dialectique insoluble entre le sujet et l'objet et forme une situation contradictoire et ambivalente : le thérapeute et le client sont à la fois sujets d'un événement intersubjectif et objets de l'autoréflexion ou l'introspection du vécu subjectif. La méthode dialectique est une possibilité pour ne pas éliminer des contradictions, mais pour les faire productives pour le processus de connaissance. La raison pourquoi la méthode dialectique soit de l'intéresse spéciale pour la science psychothérapeutique est la situation psychothérapeutique, dans laquelle le sujet et l'objet ne peuvent pas être considérés séparément. Plutôt la relation entre le thérapeute et le client forme une situation contradictoire et ambivalente et demande une compréhension dialectique.

La correspondance recherchée entre le concept de science de la psychothérapie et le concept général de science repose sur une triple acceptation :

1. la réflexion et l'autoréflexion doivent valoir comme des méthodes qualitatives de reconnaissance de la subjectivité et l'intersubjectivité ;

2. l'individualité doit être préservée malgré les généralisations du processus scientifique ;

3. des structures alogiques, telles que les contradictions, les antinomies et les ambivalences, sont reconnues comme des facteurs productives du processus scientifique. 
La méthode de l'autoréflexion respectivement de l'introspection du vécu subjectif et la réflexion de la relation entre le psychothérapeute et le client dans la situation psychothérapeutique sont caractéristique pour la science psychothérapeutique et constituent leur l'autonomie. Par ce multiple et commun processus réfléchissant du thérapeute et du client, qui sert non seulement à la guérison mais encore à la création de connaissance, la science psychothérapeutique se distingue fondamentalement de la médecine et de la psychologie clinique. À mon avis, il n'y a donc aucune raison pour qu'on attribue à la science psychothérapeutique un statut scientifique différent qu'à la médicine et à la psychologie.

\section{L'auteur}

Markus Erismann, Dr. phil., a fait de 1983 à 1989 des études de philosophie et de germanistique à l'université de Zurich et a présenté sa thèse de doctorat et 1995/96 au séminaire philosophique de l'université de Zurich. Les points forts de son travail sont la théorie de la connaissance et la méthodologie.

\section{Contact}

Dr. Markus Erismann

Säntisstrasse 2

8008 Zürich

E-Mail : markus.erismann@bluewin.ch 